\title{
Rectifier Design Using Distributed Greinacher Voltage Multiplier for High Frequency Wireless Power Transmission
}

\author{
Joonwoo Park ${ }^{1} \cdot$ Youngsub $\mathrm{Kim}^{1} \cdot$ Young Joong Yoon $^{1, *} \cdot$ Joonho $\mathrm{So}^{2} \cdot$ Jinwoo $\mathrm{Shin}^{2}$
}

\begin{abstract}
This paper discusses the design of a high frequency Greinacher voltage multiplier as rectifier; it has a greater conversion efficiency and higher output direct current (DC) voltage at high power compared to a simple halfwave rectifier. Multiple diodes in the Greinacher voltage multiplier with distributed circuits consume excited power to the rectifier equally, thereby increasing the overall power capacity of the rectifier system. The proposed rectifiers are a Greinacher voltage doubler and a Greinacher voltage quadrupler, which consist of only diodes and distributed circuits for high frequency applications. For each rectifier, the RF-to-DC conversion efficiency and output DC voltage for each input power and load resistance are analyzed for the maximum conversion efficiency. The input power with maximum conversion efficiency of the designed Greinacher voltage doubler and quadrupler is 3 and $7 \mathrm{~dB}$ higher, respectively, than that of the halfwave rectifier.
\end{abstract}

Key Words: Conversion Efficiency, Greinacher Voltage Multiplier, Rectifier, Schottky Diode, Wireless Power Transfer.

\section{INTRODUCTION}

Wireless power transmission (WPT) technology is being spotlighted as high power transmission [1]. Short range transmission has been researched by studies on inductive coupling and resonant coupling methods. However, the transfer ranges of these technologies are limited to a few centimeters to a few meters [2]. Recently, long range WPT technology using microwave radiation methods has been investigated on the strength of advances in high power radio-frequency (RF) sources and the Schottky diode [3, 4]. Unlike energy harvesting, which converts a small signal to direct current (DC) power, WPT deals with high power at the receiving system and the operating frequency is generally high to obtain a high gain for the antenna system [5]. Thus, the design para- meters of a rectifier for use in a WPT system at high frequency region differs from those use for low frequency or baseband rectifiers. Typically, a Schottky barrier diode is used for a RF rectifier due to its fast switching speed [6]. However, as the frequency increases, the maximum breakdown voltage of the Schottky diode is reduced. Consequently, this leads to a low output DC voltage as well as a low system power capacity [7]. Furthermore, a typical WPT system consists of an antenna array that delivers high power to the rectifier circuit. Therefore, designing a high frequency rectifier system with greater power capacity that can produce both high output DC voltage and high conversion efficiency at a high power is the key factor in the fabrication of the receiving system of a high frequency WPT system.

This paper is focused on improving the power capacity of

Manuscript received January 28, 2014 ; Revised March 10, 2014 ; Accepted March 10, 2014. (ID No. 20140128-003J)

${ }^{1}$ Department of Electrical and Electronic Engineering, Yonsei University, Seoul, Korea.

${ }^{2}$ Agency for Defense Development, Deajeon, Korea.

"Corresponding Author: Young Joong Yoon (e-mail: yjyoon@yonsei.ac.kr)

This is an Open-Access article distributed under the terms of the Creative Commons Attribution Non-Commercial License (http://creativecommons.org/licenses/by-nc/3.0) which permits unrestricted non-commercial use, distribution, and reproduction in any medium, provided the original work is properly cited.

(C) Copyright The Korean Institute of Electromagnetic Engineering and Science. All Rights Reserved. 
the rectifier system by using a Greinacher voltage multiplying circuit. This voltage multiplying circuit is made up of many lumped capacitors that are put between diodes [7]. However, the use of a lumped capacitor is difficult at high frequency due to its self-resonant frequency (SRF). The use of a Greinacher voltage multiplier circuit at high frequency first requires analysis of the $\mathrm{I}-\mathrm{V}$ characteristics of the Schottky barrier diode.

Every simulation and measurement in this paper is conducted using Avago Technologies HSMS-8101 and HSMS8202 Schottky barrier diode. The substrate is Taconic TLC30-0200, which has a permittivity of 3.0 and 0.003 of tangent loss at $10 \mathrm{GHz}$. A single tone signal in the $\mathrm{X}$-band is used as the experimental frequency.

\section{Rectifier with Multiple Diodes}

A rectifier converts time-varying power to $\mathrm{DC}$ power. One of the figures of merit of the RF rectifier is its RF-to-DC conversion efficiency, which is determined by following equation [1]:

$$
\eta_{\mathrm{RF}-\mathrm{to}-\mathrm{DC}}=\frac{V_{\mathrm{DC}}^{2} / R_{\mathrm{LOAD}}}{P_{\mathrm{in}}}
$$

The rectification process in the high power RF band differs from a baseband rectifier. First, most of the diodes used in RF rectifiers are Schottky diodes. The Schottky diode has a fast enough switching speed to rectify an RF signal. However, the Schottky diode has a low peak inverse voltage (PIV), so it is not practical for use in high power applications [7]. Although the Schottky diode has a low PIV and worse power handling capacity than a PN junction diode, its fast switching speed is essential for an RF rectifying system.

Secondly, the matching point varies with the input power. Power source impedance should equal the complex conjugate of the input impedance of the RF circuit. However, since the input impedance of these nonlinear circuits, including the Schottky diode, significantly changes with the input signal level, obtaining a conjugate impedance match is difficult for all input power in the WPT system. Typically, these circuits are designed for a specific value of input power level, mostly the primary operating input power level expected for the circuit [3]. Thus, the choice of an appropriate input power is a very important design factor. An input power range that exceeds the desired RF-to-DC conversion efficiency is another figure of merit of a large signal rectifier. Each element in a WPT system gets a different power due to nonuniformity of the main lobe of transmitter; therefore, the center of the receiving array and the edge of the receiving array get very different power.

Fig. 1 shows the maximum load DC voltage and conversion efficiency for a Greinacher voltage doubler versus the load resistance for each input power. This simulation is conducted under ideal input and output matching. The harmo-

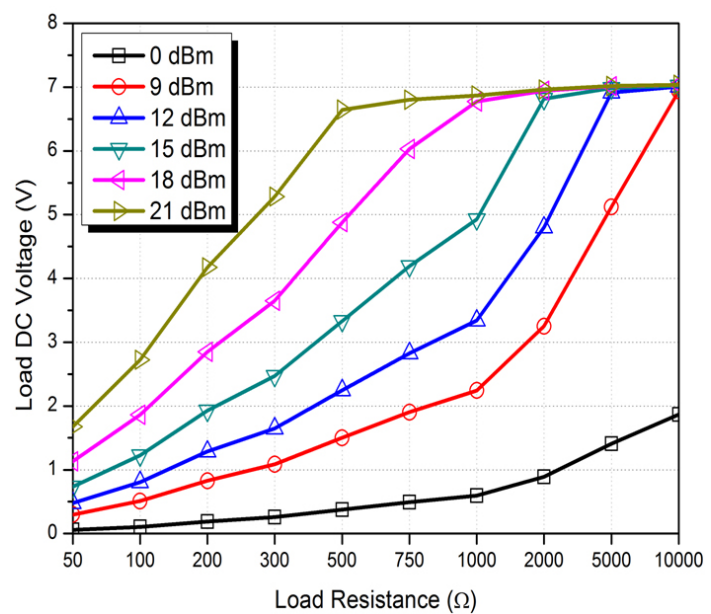

(a)

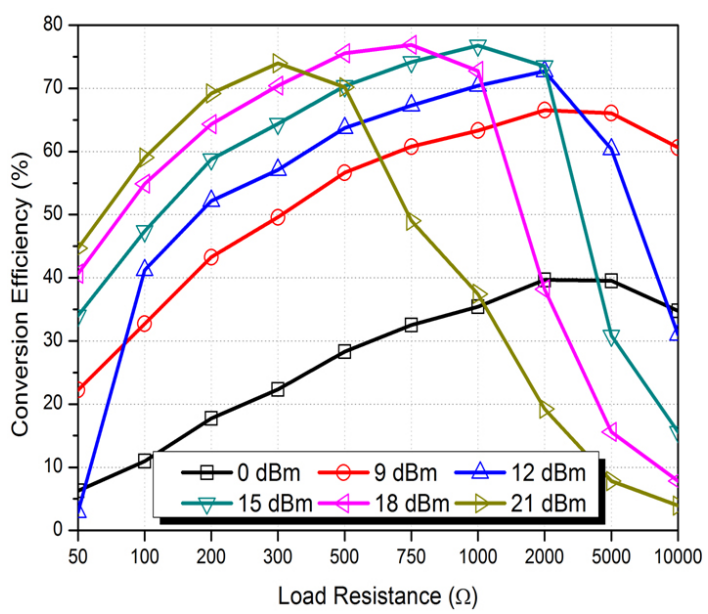

(b)

Fig. 1. Simulated maximum output DC voltage (a) and convertsion efficiency (b) of Greinacher voltage doubler versus load resistance using ideal circuit components.

nic generated by the nonlinear Schottky diode is not considered. As shown in Fig. 1(a), the output DC voltage produced by high load resistance is limited by the breakdown effect of the Schottky diode. In this paper, the use of the Avago HSMS-8102 Schottky diode results in a $7.0 \mathrm{~V}$ of saturated output DC voltage for the Greinacher voltage doubler from $9 \mathrm{dBm}$ of input power. However, the load resistance for each saturation point of input power differs from each other, as shown in Fig. 1. Thus, the load resistance and input power must be tuned for the best performance of a given WPT system.

\section{High Frequency Rectification Process}

Fig. 2 shows a conventional halfwave rectifier and Greinacher voltage multipliers. These conventional circuits contain one or more lumped capacitor. However, the use of a lumped capacitor is difficult at high frequency due to its SRF. Hence, the high frequency rectifier needs an equivalent 


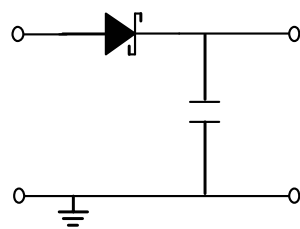

(a)

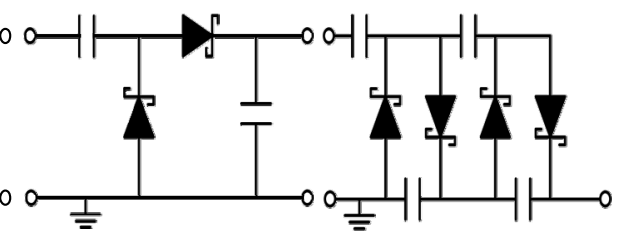

(b) (c)
Fig. 2. Halfwave rectifier and Greinacher voltage multiplier using Schottky diode. (a) Halfware, (b) doubler, and (c) quadrupler.

circuit for the lumped capacitor. Some of the lumped capacitors can also be omitted at high frequency.

Lumped capacitors are classed as shunt capacitors and series capacitors. Shunt capacitors are omitted by the $\mathrm{I}-\mathrm{V}$ characteristics of the Schottky diode. In general, for the rectifier that rectifies only a single tone signal, the power series expansion method is used to obtain the output current. The output current $y(t)$ can be expressed as Eq. (2). The constants $k_{1}, k_{2}, \cdots$ can be obtained by Eq. (3) for a specific forward bias voltage [3].

$$
\begin{aligned}
& \mathrm{y}(\mathrm{t})=k_{1} \cos \omega t+k_{2} \cos ^{2} \omega t+\cdots \\
& \mathrm{k}_{1}=\frac{1}{1 !} \frac{\partial y(x)}{\partial x}_{x=V_{d}} ; k_{2}=\frac{1}{2 !}{\frac{\partial^{2} y(x)}{\partial x^{2}}}_{x=V_{d}}
\end{aligned}
$$

The role of the shunt capacitor of the rectifier that uses a PN junction diode at a low frequency is charging, and for the mixer, the role of the shunt capacitor is as an envelope detector or low pass filter (LPF). For the rectifier using the Schottky diode, the charging mechanism is not needed due to its linear $\mathrm{I}-\mathrm{V}$ characteristic in the operating region.

Eq. (2) can be modified as follows [8]:

$$
\cos ^{\mathrm{n}} \theta=\frac{2}{2^{n}} \sum_{k=0}^{(n-1) / 2}\left(\begin{array}{l}
n \\
k
\end{array}\right) \cos ((n-2 k) \theta)
$$

for odd $n$

$$
\begin{gathered}
\cos ^{n} \theta=\frac{1}{2^{n}}\left(\begin{array}{c}
n \\
n / 2
\end{array}\right)+\frac{2}{2^{n}} \sum_{k=0}^{(n-2) / 2}\left(\begin{array}{l}
n \\
k
\end{array}\right) \cos ((n-2 k) \theta) \\
\text { for even } n
\end{gathered}
$$

Eq. (4) shows that the output current of the Schottky diode is divided into a DC part and a harmonic part. If LPF is used to eliminate this time varying power (that is, the harmonic termination network), only DC power remains; thus, the rectification process occurs. Fig. 3 shows the substitution of the harmonic termination network for a shunt capacitor [9].

The equivalent circuit model for a series capacitor is the high pass filter (HPF). However, the series capacitor used in the Greinacher voltage multiplier circuit acts as both an HPF and also a DC block (DCB) for DC bias. That is, unlike the shunt capacitor, which acts as a simple LPF, the
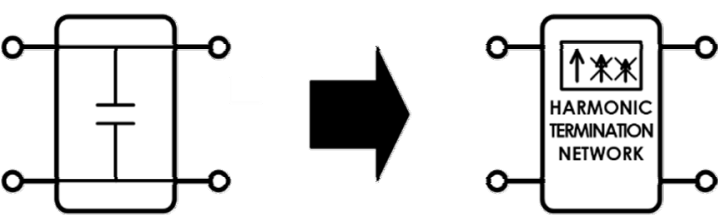

Fig. 3. Elimination process of a shunt lumped capacitor.

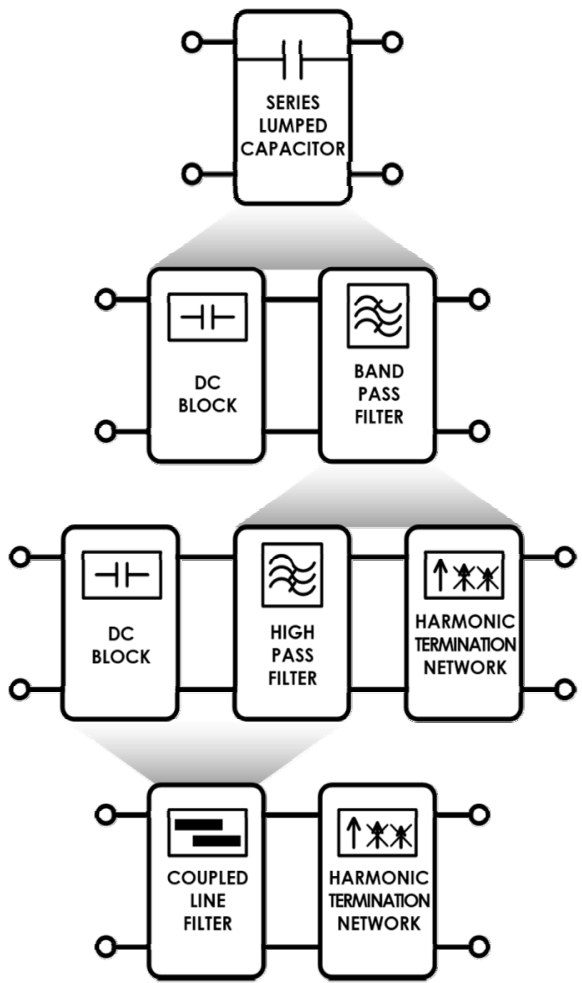

Fig. 4. Elimination process of a series lumped capacitor.

series capacitor acts as a charging capacitor. Moreover, for the series capacitor at the input network, the operating frequency surpasses this and should block higher order harmonics as well as the DC at the same time. Therefore, the series capacitor should act as a band pass filter (BPF) and a $\mathrm{DCB}$. However, realizing both the $\mathrm{BPF}$ and $\mathrm{DCB}$ at the same time is difficult, especially at a high frequency. Therefore, in this paper, the high order harmonics are terminated by an additional input harmonic termination network. As a result, the series capacitor acts as an HPF and a DCB. An additional network, such as a harmonic termination network, is also added to the HPF to realize BPF. HPF is realized using a simple coupled line filter, which also acts as a DCB [10]. These procedures are summarized in Fig. 4.

\section{High Frequency Greinacher Voltage Multiplier}

The designed Greinacher voltage multipliers are shown in Figs. 5 and 6. The input network of these rectifiers consists 
of a matching network and an input harmonic termination network. There is a via at the input harmonic termination network of the halfwave rectifier [3]. The role of this via is to eliminate the reflected DC harmonic caused by the nonlinear characteristics of the Schottky diode. However, for the Greinacher voltage multipliers, unlike the halfwave rectifier, the $\mathrm{DCB}$ moves from the foremost of the input network to the very end of the input network; that is, right in front of the diode. This is because the DCB act as an HPF, as shown in Fig. 4. This DCB is designed for passing the operating frequency and it blocks DC. Input high order harmonics are eliminated at the input harmonic termination network. Unlike the input harmonic termination network in a halfwave rectifier, the Greinacher voltage multiplier does not need a via through the ground. The reflected DC harmonic caused by the diode is blocked by a coupled line filter right in front of the diode. This makes a simpler input network than that of the halfwave rectifier. The DCB at the foremost of the input network is needed to prevent the via from affecting the receiving antenna or the signal source.

The conventional Greinacher voltage doubler and quadrupler are shown in Fig. 2(b) and (c) [11]. A high frequency, capacitorless Greinacher voltage doubler can be designed by replacing the series capacitance with $\mathrm{HPF}$ and $\mathrm{DCB}$, and the shunt capacitance with LPF. The output network of the designed rectifier consists of an output harmonic termination network and a load resistance. Since the input matching network is dramatically changed by the output network, the output network is the most important. Notably, the fundamental frequency must be eliminated at the output network. The fundamental frequency component is predominant when the input power is high. If the fundamental frequency and other higher order harmonics are not eliminated, the output DC voltage will be degraded by ripple and the DC voltage also cannot be measured. The output harmonic caused by the diode must be eliminated as near as possible to the diode. The shorter the $L_{7}$ shown in Fig. 5, the better. However, it cannot be zero. For this design, the length of $L_{7}$ is $1 \mathrm{~mm}$. If the harmonic is well eliminated, the length of $L_{9}$ does not affect the circuit impedance since the input impedance to the load resistance becomes infinite, except for DC. However, in reality, eliminating every harmonic is impossible; thus, the shorter the $L_{8}$, the better, also.

The output network of the Greinacher voltage quadrupler consists of an output harmonic termination network and a load resistance. Like the Greinacher voltage doubler, the shorter the $L_{12}$ shown in Fig. 6, the better. The length of $L_{10}$ in Fig. 6 is determined by the length of the coupled line filter. There should be a harmonic termination network at $L_{10}$. However, this harmonic termination network is negligible since every harmonic is terminated in front of the load resistance. Tables 1 and 2 show that as more diodes are connected, both the optimal load resistance and the optimal input power increase. The optimal load resistance for the halfwave rectifier in this paper is $750 \Omega$.

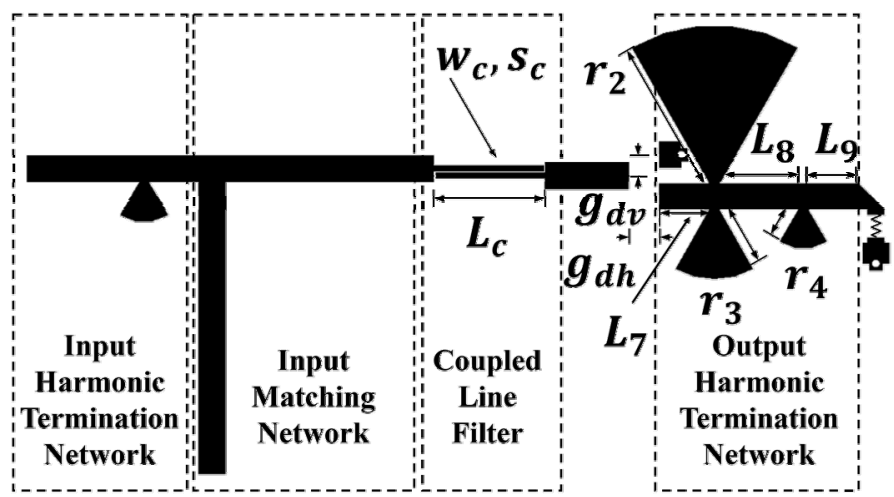

Fig. 5. The designed high frequency Greinacher voltage doubler.

Table 1. Specific values of the designed high frequency Greinacher voltage doubler

\begin{tabular}{cc|cc}
\hline Parameter & Value & Parameter & Value \\
\hline$r_{2}$ & $7.50 \mathrm{~mm}$ & $g_{d b}$ & $1.50 \mathrm{~mm}$ \\
$r_{3}$ & $3.27 \mathrm{~mm}$ & $g_{d v}$ & $1.00 \mathrm{~mm}$ \\
$r_{4}$ & $1.80 \mathrm{~mm}$ & $L_{7}$ & $2.00 \mathrm{~mm}$ \\
$w_{c}$ & $0.25 \mathrm{~mm}$ & $L_{8}$ & $3.00 \mathrm{~mm}$ \\
$L_{c}$ & $5.36 \mathrm{~mm}$ & $L_{9}$ & $2.00 \mathrm{~mm}$ \\
$s_{c}$ & $0.087 \mathrm{~mm}$ & $R_{\mathrm{LOAD}}$ & $1,000 \Omega$ \\
\hline
\end{tabular}

Table 2. Specific values of the designed high frequency Greinacher voltage quadrupler

\begin{tabular}{cc|cc}
\hline Parameter & Value & Parameter & Value \\
\hline$r_{2}$ & $7.50 \mathrm{~mm}$ & $L_{9}$ & $1.00 \mathrm{~mm}$ \\
$r_{3}$ & $3.27 \mathrm{~mm}$ & $L_{10}$ & $5.36 \mathrm{~mm}$ \\
$r_{4}$ & $1.80 \mathrm{~mm}$ & $L_{11}$ & $1.00 \mathrm{~mm}$ \\
$L_{7}$ & $2.26 \mathrm{~mm}$ & $R_{\mathrm{LOAD}}$ & $2,000 \Omega$ \\
$L_{8}$ & $1.00 \mathrm{~mm}$ & - & - \\
\hline
\end{tabular}

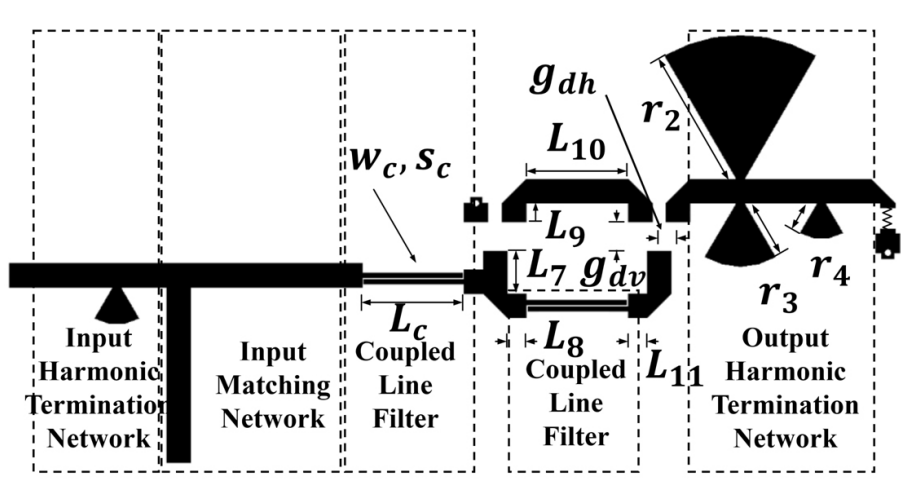

Fig. 6. The designed high frequency Greinacher voltage quadrupler.

\section{Measurement and Results}

Fig. 7(a) shows the output DC voltage for the three types 


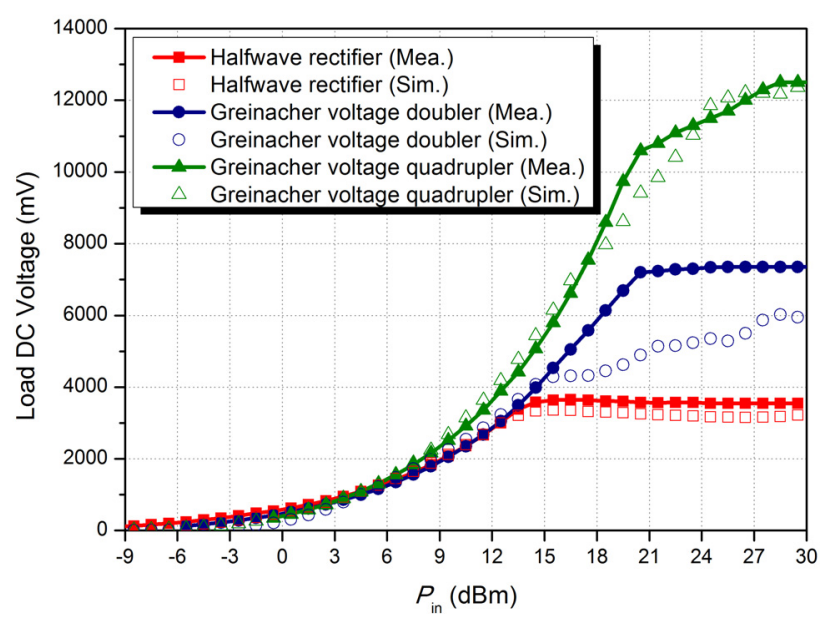

(a)

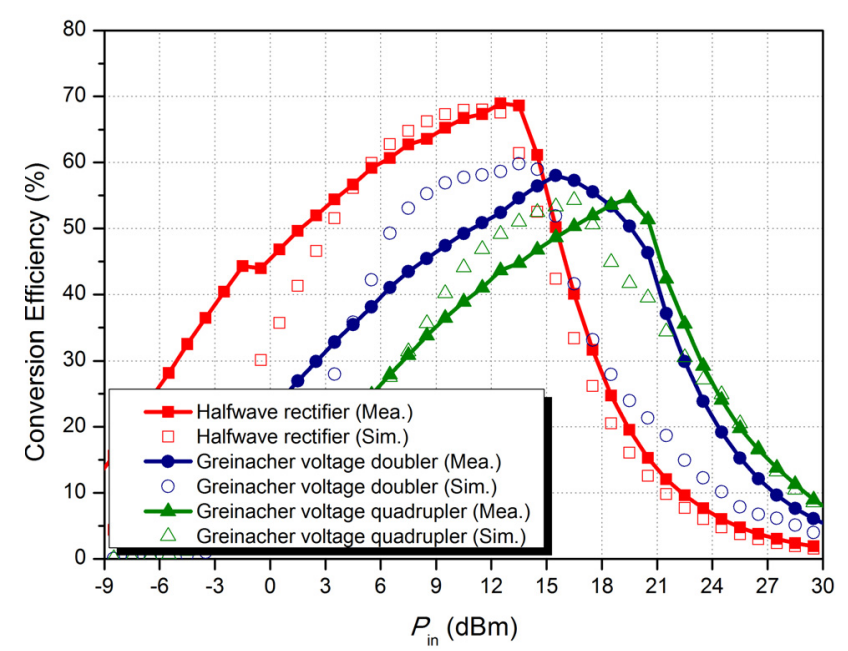

(b)

Fig. 7. Measured and simulated load DC voltage (a) and conversion efficiency $(b)$ of each rectifier.

of rectifiers, while Fig. 7(b) shows the conversion efficiency. As diodes double, the power handling capacity also increases by $3 \mathrm{~dB}$ and this is shown in both Fig. 7(a) and (b). The peak point of conversion efficiency occurs when the input power is 13,16 , and $20 \mathrm{dBm}$ for the halfwave rectifier, Greinacher voltage doubler, and quadrupler, respectively. The breakdown voltage is the main parameter that controls the power handling capacity of the rectifier and it can be increased by placing multiple diodes. Multiple diodes consume excited power equally; therefore, the overall power handling capacity of the rectifier system increases. As the power handling capacity is increased by $3 \mathrm{~dB}$ for each other, the peak point of conversion efficiency also varies by $3 \mathrm{~dB}$, as shown in Fig. 7(b). However, the Greinacher voltage multiplier does not always multiply the output voltage but multiplies the saturated voltage. This phenomenon is seen in Fig. 7(a) and is due to the DC component generated from the Schottky diode. For the halfwave rectifier, this generated DC component is eliminated by a grounded stub. However, for the Greinacher voltage multiplier, the DCB blocks the generated

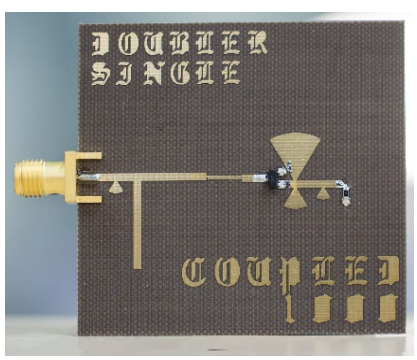

(a)

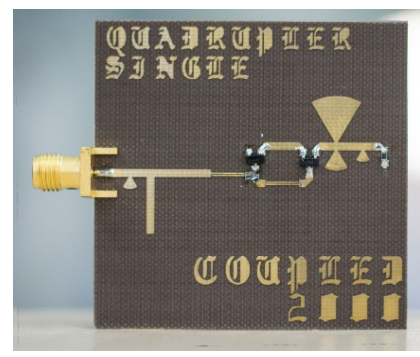

(b)
Fig. 8. Fabricated rectifiers. (a) Doubler, (b) quadrupler.

DC component, thereby lowering the self DC bias in front of the diodes.

The results show that a halfwave rectifier, which is a reference rectifier, shows $3.55 \mathrm{~V}$ of saturated voltage and $69 \%$ of maximum conversion efficiency at $12.5 \mathrm{dBm}$ of input power. The optimal load resistance for a halfwave rectifier is $745 \Omega$. The Greinacher voltage doubler shows $7.35 \mathrm{~V}$ of saturated output DC voltage and shows $58 \%$ of maximum conversion efficiency at $15.5 \mathrm{dBm}$ of input power, which is $3 \mathrm{~dB}$ higher than that of the halfwave rectifier. The optimal load resistance for the Greinacher voltage doubler is $997 \Omega$. The Greinacher voltage quadrupler shows $12.50 \mathrm{~V}$ of saturated output DC voltage and shows $55 \%$ of maximum conversion efficiency at $19.5 \mathrm{dBm}$, which is $7 \mathrm{~dB}$ higher than that of the halfwave rectifier. The optimal load resistance for the Greinacher voltage quadrupler is 1,950 $\Omega$. Resistance values are measured values and fabricated circuits are shown in Fig. 8. Degradation of the maximum conversion efficiency is due to a generated DC component.

\section{CONCLUSION}

The use of the distributed Greinacher voltage multiplier increases the power handling capacity by about $3 \mathrm{~dB}$ by doubling the number of diodes. As more rectifiers are increasingly doubled, the optimal resistance for maximum conversion efficiency is increased. The research presented here indicates that the proposed distributed Greinacher voltage multiplier can be applied to high power WPT systems. The measurement results are similar to method of moment simulation results, and the tendency is pronounced.

\section{REFERENCES}

[1] W. C. Brown, "The history of power transmission by radio waves," IEEE Transactions on Microwave Theory and Techniques, vol. 32, no. 9, pp. 1230-1242, Sep. 1984.

[2] B. Wang, K. H. Teo, T. Nishino, W. Yerazunis, J. Barnwell, and J. Zhang, "Experiments on wireless power transfer with metamaterials," Applied Physics Letters, vol. 98, no. 25, p. 254101, Jun. 2011.

[3] A. Boaventura, A. Collado, N. B. Carvalho, and A. Georgiadis, "Optimum behavior," IEEE Microwave Magazine, vol. 14, no. 2, pp. 26-35, Mar. 2013. 
[4] K. Takayama, S. Hiramatsu, and M. Shiho "CW 100 MW microwave power transfer in space," in Proceedings of the Particle Accelerator Conference, San Francisco, CA, 1991, pp. $2625-2627$.

[5] S. S. Mohammed, K. Ramasamy, and T. Shanmuganantham, "Wireless power transmission: a next generation power transmission system," International Journal of Computer Applications, vol. 1, no. 13, pp. 100-103, 2010.

[6] R. Ludwig and P. Bretchko, RF Circuit Design: Theory and Application. Upper Saddle River, NJ: Prentice Hall, 2000.

[7] I. Batarseh, Power Electronic Circuits. Hoboken, NJ: John Wiley \& Sons, 2004.

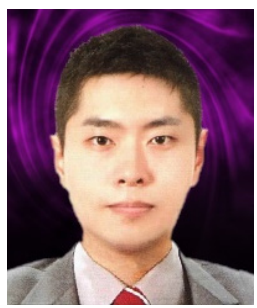

Joonwoo Park received the B.S. degree in electronic engineering from Yonsei University, Seoul, Korea in 2012 and received the M.S. degree in electrical and electronic engineering from Yonsei University, Seoul, Korea, in 2014. His research interests include optics, high power applications and electronic warfare.

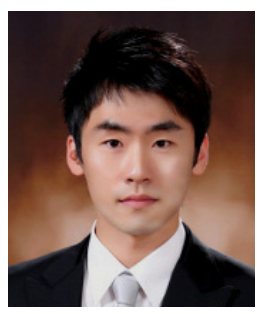

Youngsub Kim received the B.S. degree in electronic engineering from Dongguk University, Seoul, Korea in 2010 and received the M.S. degree in electrical and electronic engineering from Yonsei University, Seoul, Korea, in 2012. Since 2012, he is currently working toward the $\mathrm{Ph}$.D. degree at Yonsei University. His research interests include beam synthesis of arrays, reflector antenna, and wireless power transfer.

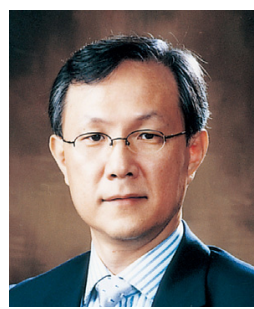

Young Joong Yoon (M'93) received the B.S. and M.S. degrees in electronic engineering from Yonsei University, Seoul, Korea, in 1981 and 1986, respectively, and the Ph.D. degree in electrical engineering from the Georgia Institute of Technology, Atlanta, in 1991. From 1992 to 1993, he was a Senior Researcher with the Electronics and Telecommunications Research Institute (ETRI), Daejeon, Korea. In 1993, he joined the faculty of Yonsei University, where he is currently a Professor with the Department of Electrical and Electronics Engineering. From 2011 to 2012, he was a president at the Korean Institute of Electromagnetic Engineering \& Science (KIEES). His research interests are antennas, $\mathrm{RF}$ devices, and radio propagations.
[8] D. Zwillinger, Standard Mathematical Tables and Formulae, 31st ed. Boca Raton, FL: Chapman \& Hall/CRC, 2003.

[9] J. Park, Y. Kim, W. C. Choi, Y. J. Yoon, J. Shin, J. So, and H. Kim, "X-band rectifier design using harmonic termination network," in Proceedings of the Korean Institute of Electromagnetic Engineering and Science Summer Workshop, Jeju, Korea, 2013, p. 226.

[10] D. M. Pozar, Microwave Engineering, 3rd ed. Hoboken, NJ: John Wiley \& Sons, 2005.

[11] R. L. Boylestad and L. Nashelsky, Electronic Devices and Circuit Theory, 9th ed. Upper Saddle River, NJ: Pearson Education International, 2006.

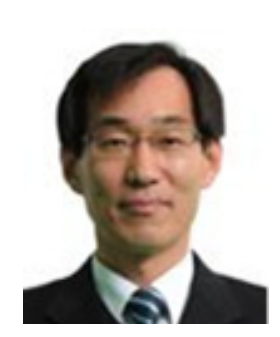

Joonho So received his B.S. degree, the M.S. degree and $\mathrm{Ph} . \mathrm{D}$. degree in electrical engineering from University of Seoul, Seoul, Korea in 1988, 1990, and 2006, respectively. Since 1990, he has been working as a researcher at Agency for Defense Development. His current interests are the numerical modeling and analysis of active array, high power microwave and $\mathrm{THz}$ system.

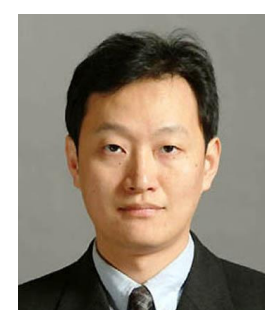

Jinwoo Shin received the M.S. degree in electrical engineering from the University of Seoul, Korea, in 1997. Since 1997, he has been working as a researcher at Agency for Defense Development. His current research interests are the numerical modeling and analysis for wireless power transmission, active array antenna and high power vacuum tubes. 